\title{
PROPRIETES BIOLOGIQUES
}

\section{Données récentes sur les propriétés immunoallergologiques des protéines laitières}

\author{
par \\ Anne Moneret-Vautrin*, G. Humbert**, C. Alais** \\ J. P. GRILliat*
}

Rés u mé

Le lait, par ses protéines, est le trophallergène le plus incriminé dans les allergies alimentaires de l'enfant, et le cinquième chez l'adulte. Son pouvoir immunogène est démontré par la constance d'une réponse immunologique chez le jeune nourrisson. Due au caractère hétérologue de ses protéines, l'antigénicité du lait existe à trois niveaux : celui des protéines natives, celui des produits de la digestion, celui des produits modifiés par la technologie : condensation, évaporation, conservation à l'état de poudre, chauffage, etc. Des travaux modernes remettent en cause la conception classique de l'importance des réactions de Maillard dans l'antigénicité. Les circonstances favorisant la sensibilisation aux protéines du lait ont trait aux particularités immunologiques de l'organisme, à celles de l'apport alimentaire, aux voies de sensibilisation, au rôle protecteur de l'allaitement, au rôle aggravant ou déclenchant de certaines affections intercurrentes. La multiplicité des diagnostics possibles lorsqu'il existe des symptômes à l'ingestion de lait, la difficulté de mise en

* Service de Médecine " D » (Pr J. P. Grilliat, Pr Ag. D. A. Moneret-Vautrin), Médecine Interne et Immunoallergologie, C.H.U. de Nancy-Brabois, route de Neufchâteau - 54500 Vandœuvre-lès-Nancy.

** Laboratoire de Biochimie Appliquée ( $\operatorname{Pr}$ C. Alais), Université de Nancy I. B.P. 239 - 54506 Vandœuvre-lès-Nancy. 
évidence de la réponse immuno-allergique ne doivent pas faire oublier que le problème est susceptible de se déplacer des protéines natives à des composés encore mal appréciés quant à leurs potentialités immunogènes, créées par les nouvelles technologies, ou à de possibles sensibilisations à des haptènes fixés sur ces protéines, contaminant le lait.

\section{Mots clés}

Lait - Bêtalactoglobuline - Alphalactalbumine - Caséine.

Antigénicité - Allergénicité - Modifications par digestion, par technologie.

Atopie - Apport excessif - Voies de sensibilisation - Allaitement.

Tests immunoallergologiques.

Titre abrégé

Immunoallergologie des protéines laitières.

\section{Sum mary}

\section{MODERN APPROACH OF IMMUNOALLERGIC PROPERTIES OF MILK PROTEINS}

Milk is the commonest allergen in pediatric food allergy (the fifth in adults) Immunologic potency is prooved by the well established immunologic response in normal infants.

Heterologous cow's milk proteins exhibit antigenicity at three levels: native proteins, proteins modified by digestion, and proteins modified by technological processes: condensation, evaporation, drying, heating etc. Conceptions which are now prevailing question the allegated implication of Maillard's reactions in antigenicity. Numerous circumstances favourize hypersensitivity to cow's milk: immunologic peculiarities of the organism, protective role of breastfeeding, average of daily intakes, routes of sensitization, responsibility of arising illnesses like viral infections. The authors insist on the difficulties of the diagnosis of intolerance to cow's milk proteins a which require an immunoallergologic study. They emphasize the possibility of growing allergenicity, depending on new technological processes, and of sensitization to contaminating haptens, reacting with proteins.

\section{Key words}

Milk - Betalactoglobulin - Alphalactalbumin - Casein.

Antigenicity - Allergenicity - Modification by digestion, by technology.

Atopy - Excessive intake - Routes of sensitization - Breast-feeding Immunoallergic tests.

Run title

Immunoallergology of milk proteins. 


\section{INTRODUCTION}

Parmi les aliments, le lait d'origine bovine constitue une des principales sources d'allergènes alimentaires : par ordre de fréquence, il est le premier trophallergène chez l'enfant, le cinquième chez l'adulte, après le poisson, l'œuf, les crustacés, le céleri. Les tableaux cliniques les plus fréquents sont, classiquement, de façon prédominante, digestifs : douleurs abdominales, diarrhée, vomissements. Chez l'adulte, il s'agit de symptômes fonctionnels, sans altération de la muqueuse digestive. Chez le nourrisson, ils traduisent une atteinte de la muqueuse du grêle allant jusqu'à l'atrophie villositaire totale (Hill et al., 1979). Les symptômes extra-digestifs comportent le choc anaphylactique, l'urticaire ou l'œdème de Quincke, l'asthme, l'eczéma, et d'autres tableaux plus rares. Les points d'intérêt actuels sont :

1. Une meilleure connaissance des problèmes complexes de l'antigénicité des différentes protéines responsables de l'allergie alimentaire au lait.

2. Une meilleure évaluation des circonstances favorisant la sensibilisation.

3. Un affinement des méthodes de diagnostic.

\section{L'ANTIGENICITE DES PROTEINES}

L'antigénicité du lait de vache est liée au caractère hétérologue des protéines qui le constituent. Son importance est démontrée par le fait que, constamment, chez le nouveau-né normal, lui correspond une réponse immunologique, dans les 12 premiers jours de l'alimentation lactée. Les anticorps, de classe IgG, mis en évidence par différentes méthodes (précipitation, fixation du complément, hémagglutination) atteignent leur valeur maximale dès l'âge de 3 mois, restent stables jusqu'à 18 mois, puis déclinent (Lippard et al., 1936; Gunther et al., 1960; Kletter et al., 1971). Cette réponse anticorps est physiologique : elle correspond au passage facile des protéines lactées à travers la muqueuse intestinale, perméable dans le jeune âge.

Le pouvoir immunogène des protéines du lait de vache est donc accentué. Il doit être étudié à 3 niveaux : celui des protéines natives, celui de leurs produits de digestion, celui des protéines modifiées par la technologie, allant du simple chauffage à des traitements plus complexes.

\section{Antigénicité des protéines natives}

Les protéines natives sont une vingtaine environ : les $4 / 5^{\mathrm{e}}$ sont représentés par les caséines; le reste, par les protéines solubles du 
TABLEAU 1 - TABLE 1

Rôles respectifs des différentes protéines du lait dans l'allergie alimentaire au lait (étude par test de dégranulation des basophiles humains)

Relative frequency of different proteins in food allergy to milk (studied by the human basophil degranulation test)

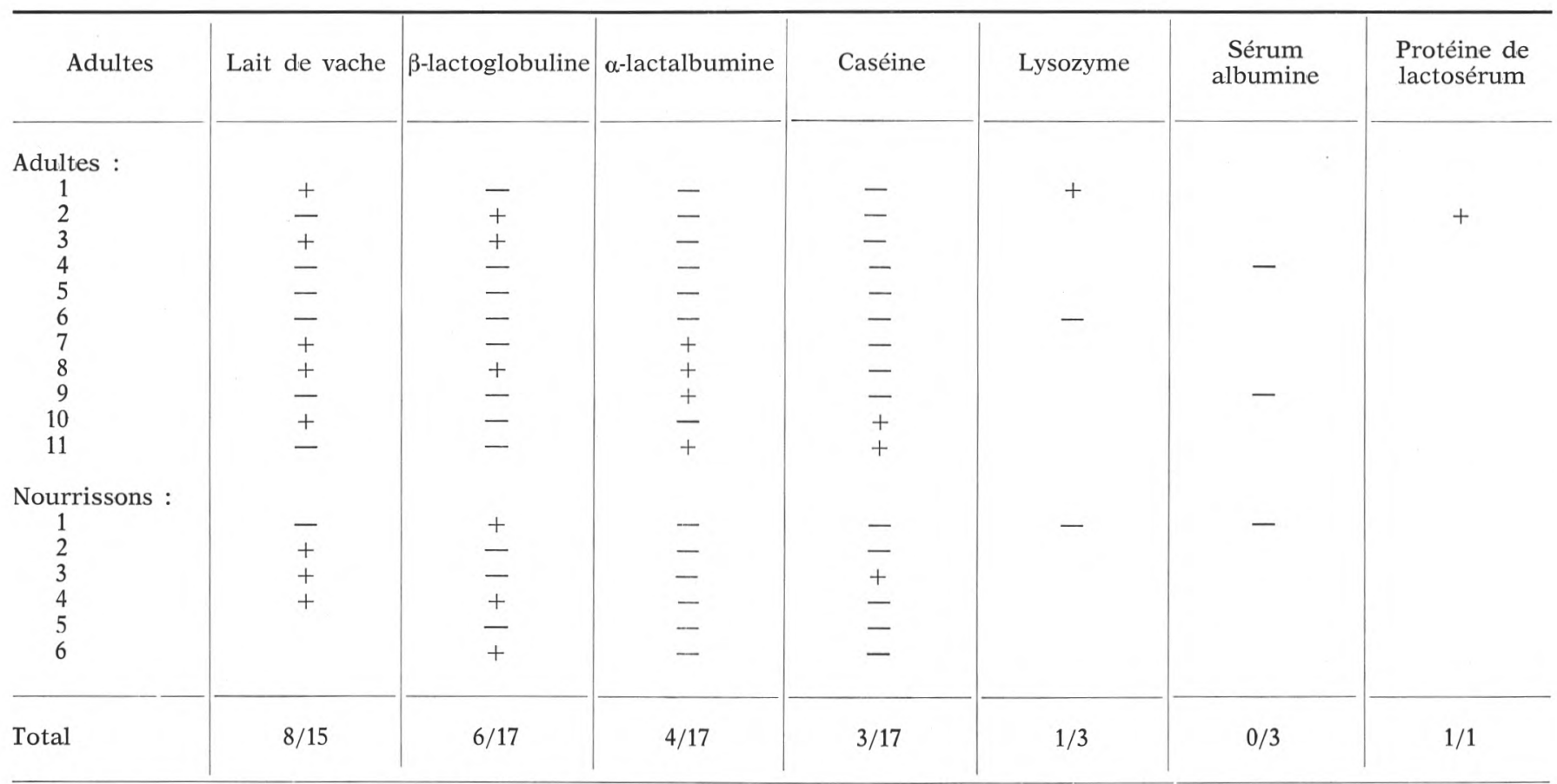

L'antigène majeur est la $\beta$-lactoglobuline, suivie par l' $\alpha$-lactalbumine, puis la caséine. 
lactosérum : bétalactoglobuline, alpha-lactalbumine et sérumalbumine en représentent $77 \%$, les immunoglobulines $12 \%$, les protéoses-peptones $10 \%$, les protéines enzymatiques le restant.

Une activité antigénique est démontrée pour la caséine (PM = 20000 à 24000$)$, la bétalactoglobuline ( $\mathrm{PM}=18000)$, la sérumalbumine $(P M=69000)$, l'alpha-lactalbumine $(P M=16000)$. Cette dernière présente une grande parenté avec le lysozyme $(\mathrm{PM}=14000)$ retrouvé dans le blanc d'œuf. Certains cas d'allergie alimentaire au blanc d'œuf et au lait ressortent vraisemblablement d'une sensibilité à la séquence polypeptidique commune à l'alpha-lactalbumine et au lysozyme (une réaction croisée avec les médicaments contenant du lysozyme est également possible). Chez l'homme, l'antigène majeur est, de l'avis de différents auteurs, la bétalactoglobuline. En fait, l'étude immunologique montre que fréquemment les sujets sont sensibilisés à 2 voire 3 protéines différentes (Moneret-Vautrin et Grilliat, 1979) (tab. 1). La sérumalbumine bovine (SAB) est un antigène non négligeable, mis en évidence par les tests cutanés chez les enfants allergiques au lait. Sa séquence amino-acide est connue, ainsi que la structure tri-dimensionnelle. Wahn et al., (1981), ont démontré l'existence de 4 sites antigéniques contre lesquels existent des IgE spécifiques. Contrairement au cas du poisson, l'allergénicité de la SAB dépend de la structure conformationnelle. Il n'y a pas de réactivité croisée avec l'albumine de rat ou de souris.

\section{Modifications de l'antigénicité lors de la digestion}

La digestion modifie l'antigénicité dans des sens tout à fait inverses selon les protéines considérées. Ainsi, les quatre sites spécifiques de la $\mathrm{SAB}$ sont dégradés facilement par la pepsine et la trypsine (Wahn et al., 1981). Il paraît improbable que la digestion de la $\mathrm{SAB}$ démasque d'autres sites antigéniques (Wright et Rothberg, 1971). Effectivement, il est rare que le clinicien incrimine cette protéine dans les allergies au lait. Du reste, il n'est pas fréquent d'observer une allergie croisée avec la viande de bœuf, ce qui devrait être la règle en cas de sensibilisation à la SAB.

Par contre, la pepsine et la trypsine libèrent, à partir de la bétalactoglobuline, de nouveaux antigènes, comme l'ont démontré Haddad et al., en 1979. Sur dix sujets présentant une allergie clinique au lait, seuls quatre sur dix présentaient des anticorps vis-à-vis du lait et de la bétalactoglobuline. Dans les 6 autres cas, la recherche d'anticorps vis-à-vis des produits de digestion peptique, ou peptique puis tryptique, a été positive. Il est intéressant de noter que trois de ces patients présentaient un asthme d'installation tardive, quelques heures après l'ingestion. Cependant, ces antigènes créés par la digestion ne paraissent pas avoir une grande importance pratique. 


\section{Modifications de l'antigénicité induites par la technologie}

Des modifications de l'antigénicité induites par la technologie doivent être envisagées. On sait que ces traitements induisent des modifications variées des constituants glucidiques et protéiques : dénaturation, dégradation, interactions avec formation de nouveaux types de liaisons, en particulier dues à des réactions de Maillard (Adrian, 1972). La concentration élevée en lactose et la fragilité particulière des protéines du sérum rendent les produits laitiers (poudres de lait) extrêmement sensibles à la réaction de Maillard. Ainsi, évaporation, stérilisation, condensation, traitements U.H.T., sont responsables d'un blocage de la lysine de l'ordre de 5 à $15 \%$.

Or, Berrens, en 1971, a étudié la structure chimique de nombreux allergènes, constituant les squames, les plumes, les poussières de maison, certains trophallergènes comme la tomate, le lait, l'œuf. Il a montré que ces allergènes comportent tous des composés chimiques incluant les groupes $\mathrm{N}$-substitués 1-amine-1 deoxy-2 cétose, produits par les réactions de Maillard. Il a avancé l'hypothèse que cette configuration chimique caractériserait l'allergénicité d'une molécule.

C'est pourquoi les traitements aboutissant à créer des réactions de Maillard, comme le chauffage, mais également le vieillissement des poudres de lait (Luquet et al., 1980), ont été accusés d'augmenter l'allergénicité du lait. Bleumink et Young (1968), Bleumink (1970) ont soutenu cette hypothèse, en comparant, chez des sujets sensibilisés au lait, le pouvoir réactogène cutané de la bétalactoglobuline native et de la bétalactoglobuline couplée au lactose par chauffage : celle-ci se révèle 100 fois plus réactive que la première. On peut, cependant, remarquer que l'extrapolation d'un pouvoir réactogène (vis-à-vis d'un organisme déjà sensibilisé) à un pouvoir immunogène (vis-à-vis d'une organisme neuf) présente une difficulté méthodologique.

Cette conception est actuellement remise en cause par les travaux d'Anderson et al., (1979), Campbell et Vier (1978) et de Mc Laughlan et al., (1981), fondés sur l'étude d'un modèle expérimental de sensibilisation sur le cobaye. De la comparaison de diverses préparations lactées, il ressort, au contraire, que le produit chauffé $20 \mathrm{~min}$ à $110^{\circ} \mathrm{C}$ n'a plus aucune immunogénicité. La comparaison des laits entiers et en poudre montre que ces derniers sont moins allergisants. Enfin, les hydrolysats de caséine (comme le Prégestimil) présenteraient une innocuité totale. La mise au point de nouveaux produits laitiers, appelés " autogels », nécessite le recours à de semblables modèles expérimentaux, afin d'apprécier leur pouvoir immunogène (Legrand et Paul, 1977).

Cette nouvelle conception correspond mieux aux notations cliniques. En effet, le sujet allergique au lait ne signale pas de réactions 
plus accentuées avec le lait chauffé. Bien au contraire, il incrimine davantage le lait cru. Il tolère, en général, le lait en poudre et les fromages. Lorsqu'il présente, inversement, des symptômes en relation avec la consommation de fromages fermentés, il ne s'agit pas le plus souvent d'une allergie aux protéines lactées, car il boit du lait sans incidents. Le diagnostic est une pseudo-allergie alimentaire par intolérance à la tyramine.

\section{LES CIRCONSTANCES FAVORISANT LA SENSIBILISATION AUX PROTEINES LACTEES SONT NOMBREUSES}

Elles ont trait aux particularités immunologiques de l'organisme, à celles de l'apport alimentaire, aux voies de sensibilisation, au rôle protecteur de l'allaitement, au rôle aggravant ou déclenchant de certaines affections intercurrentes.

a) L'ALLERGIE AUX PROTÉINES du lait de vache survient le plus souvent, comme toute allergie alimentaire, chez des sujets atopiques, c'est-àdire ayant une programmation génétique à élaborer une grande quantité $\mathrm{d} \operatorname{IgE}$, et à déclencher avec facilité une réponse allergique à $\mathrm{IgE}$ spécifique.

L'atopie est notée dans $85 \%$ des cas d'allergie alimentaire. Le lait étant le premier trophallergène ingéré, l'allergie au lait est l'une des réponses les plus précoces du nourrisson atopique. La maturation du système immunologique local explique l'évolution spontanément régressive, aboutissant le plus souvent à la guérison des symptômes vers l'âge de 2 ans. Il est intéressant de noter que, de nombreuses années après, on peut mettre en évidence la persistance d'anticorps $\operatorname{IgE}$ au lait, en faible quantité, en utilisant un test sensible comme le test de dégranulation des basophiles humains (TDBH) (Gérard et al., 1981), dans la moitié des cas (tab. 2). Dans certains cas, la réponse en anticorps allergiques se recrute dans les IgG STS (Short term sensitizing), mises en évidence par Parish (1982).

b) L'APPORT ALIMENTAIRE LACTÉ est souvent important : soit parce qu'il constitue le seul aliment, au début de la vie; soit en raison de conditions psychologiques (régimes amaigrissants lacto-végétariens), ou liées à la profession (consommation accrue chez les ouvriers de l'industrie laitière, etc.).

c) L'ÉTUDE DES VOIES DE SENSIBILISATION revêt un intérêt particulier. Outre la classique voie digestive, signalons l'importance de l'inhalation. Elle représente, expérimentalement, une excellente voie de sensibilisation. Elle jouerait un rôle méconnu chez le nourrisson présentant, physiologiquement, des régurgitations. Le risque serait majoré chez les nourrissons nerveux et vomisseurs. L'anaphylaxie au lait inhalé pourrait expliquer certains cas de mort subite 
TABLEAU 2 - TABLE 2

Etude de 6 cas d'allergie guérie au lait de vache : mise en évidence de la persistance des IgE spécifiques Persistance of specific IgE clinical recovery, in 6 cases of food allergy

\begin{tabular}{|c|c|c|c|c|c|c|c|c|c|c|c|}
\hline & Sexe & $\begin{array}{l}\text { Age } \\
\text { (ans) }\end{array}$ & $\begin{array}{l}\text { Signes cliniques } \\
\text { dans l'enfance }\end{array}$ & Prick & ID-R & TDBH & RAST & SL & Natif & $\operatorname{IgE}$ & $\begin{array}{c}\text { Ingestion } \\
\text { courante } \\
\text { de lait }\end{array}$ \\
\hline 1 & $\mathrm{~F}$ & 27 & Allergie digestive & n.d. & + & $\begin{array}{l}+\mathrm{CL} \\
-\mathrm{ST}\end{array}$ & & & - & 92 & oui \\
\hline 2 & $\mathrm{~F}$ & 12 & Allergie digestive & - & + & $+\mathrm{CL}$ & & & - & 286 & oui \\
\hline 3 & $\mathrm{~F}$ & 15 & $\begin{array}{c}\text { Eczéma }+ \\
\text { Allergie digestive }\end{array}$ & n.d. & + & $\begin{array}{l}-\mathrm{CL} \\
-\mathrm{ST}\end{array}$ & 0 & - & - & 272 & non \\
\hline 4 & M & 7 & $\begin{array}{c}\text { Eczéma } \\
\text { constitutionnel }\end{array}$ & - & - & $+\mathrm{ST}$ & & & & & oui \\
\hline 5 & M & 33 & $\begin{array}{c}\text { Eczéma }+ \\
\text { Allergie digestive }\end{array}$ & n.d. & n.d. & $\begin{array}{l}-\mathrm{CL} \\
-\mathrm{ST}\end{array}$ & 0 & & - & 100 & oui \\
\hline 6 & M & 25 & Allergie digestive & n.d. & 一 & n.d. & & 一 & - & 990 & oui \\
\hline \multicolumn{5}{|c|}{$\begin{array}{l}\text { Ces } 6 \text { sujets consultent pour : } \\
\text { 1. Choc anaphylactique médicamenteux. } \\
\text { 2. Pollinose. } \\
\text { 3. Oedème de Quinke. } \\
\text { 4. Rhinite allergique. } \\
\text { 5. Eczéma constitutionnel. } \\
\text { 6. Asthme et urticaire. }\end{array}$} & & $\begin{array}{l}\text { Prick } \\
\text { ID-R } \\
\text { TDBH } \\
\text { RAST } \\
\text { SL } \\
\text { Natif } \\
\text { ST } \\
\text { CL } \\
\text { n.d. }\end{array}$ & \multicolumn{5}{|c|}{$\begin{array}{l}=\text { puncture épidermique. } \\
=\text { intradermoréaction. } \\
=\text { test de dégranulation des basophiles humains. } \\
=\text { radioallergo-sorbent-test. } \\
=\text { sublingual. } \\
=\text { aliment natif. } \\
=\text { sang total. } \\
=\text { cellules lavées. } \\
=\text { non déterminé. }\end{array}$} \\
\hline
\end{tabular}


chez le nourrisson (en effet, des études épidémiologiques ont confirmé que la mort subite est 2 fois moins fréquente chez les enfants nourris au lait maternel que chez les enfants recevant un lait industriel) (Deschamps, 1978). Chez l'adulte, l'inhalation de protéines lactées est réalisée dans certaines branches de l'industrie laitière (extraction des protéines du lactosérum) ou chez les sujets manipulant fréquemment des aliments lactés à usage vétérinaire. La sensibilisation conduit à un asthme professionnel (Moneret-Vautrin et al., 1982). Une sensibilisation croisée est observable chez des techniciens de laboratoire, travaillant à la centrifugation de sang bovin.

La sensibilisation par contact cutané est exceptionnelle pour les protéines, mais il peut exister une dermite de contact aux protéines du lait chez des ouvriers des fromageries amenés à manipuler des fromages blancs de façon prolongée.

Enfin, exceptionnellement, une sensibilisation a pu être déclenchée par injections de protéines lactées à usage thérapeutique (protéines lactées utilisées autrefois par voie intra-veineuse pour le traitement de l'ulcère gastrique, et lysozyme, en forme orale ou injectable).

d) Le role de l'allaitement maternel est fondamental dans la prévention de l'allergie aux protéines de lait de vache. Le nourrisson allaité par sa mère exclusivement n'entre pas en contact avec les PLV à dose importante. Celui qui bénéficie d'une alimentation mixte (lait maternel et lait d'origine bovine) est protégé par des anticorps maternels anti-bétalactoglobulines, présents dans le colostrum. Les IgA sécrétoires contribuent à empêcher le passage de la bétalactoglobuline à travers la muqueuse du nourrisson. D'autre part, le lait maternel, si la mère boit du lait, contient des traces de bétalactoglobuline et de protéines lactées en général. On peut faire l'hypothèse que ces traces d'antigènes informent le système immunologique local du nourrisson (G.A.L.T.*), et l'orientent vers une réponse immunologique particulière de tolérance. Cette réponse fera que la prise ultérieure de lait de vache, en grande quantité, sera bien tolérée.

e) LE ROLE D'AFFECTIONS INTERCURRENTES DANS LA SENSIBILISATION DU LAIT fait l'objet d'un assez large consensus. Une gastro-entérite virale est fréquemment notée dans les 2 mois précédant les signes cliniques, et on invoque essentiellement l'altération de la muqueuse digestive. Il est moins connu que chez l'adulte des infections virales peuvent entraîner la réapparition d'une allergie alimentaire au lait

* G.A.L.T. : Gut Associated Lymphoïd Tissue. 
guérie. Nous en avons observé un cas, dû à une mononucléose infectieuse sérieuse. L'action serait une stimulation du système immunitaire provoquant une réascension des anticorps, ou une réaccentuation d'une hypersensibilité retardée.

\section{LE DIAGNOSTIC D'ALLERGIE AU LAIT RESTE DIFFICILE}

Sur le plan différentiel, il faut éliminer une intolérance au lactose, par déficit congénital ou acquis, en dissaccharidases; une fausse allergie alimentaire, par intolérance à l'histamine ou à la tyramine de fromages fermentés, de pâtes cuites, comme le gruyère, ou une intolérance au nitrite de sodium (anti-oxydant présent en quantités élevées dans des fromages importés). Il faut rechercher une allergie à un contaminant, tel que la Pénicilline dont la persistance à l'état de résidus pénicilloyl fixés sur l'albumine est remarquable, comme l'ont montré les travaux de Wahl (1980); et distinguer le problème du dégoût psychologique, provoquant des manifestations considérées comme du ressort d'une allergie digestive. Ce dernier cas peut, du reste, être dû à un conditionnement négatif né, dans l'enfance, de l'inconfort d'une authentique allergie au lait.

Les méthodes de diagnostic sont multiples et l'ordre des investigations complexes ne peut être abordé ici (Moneret-Vautrin et Grilliat, 1982). Parmi tous les tests proposés, les tests cutanés (prick-tests et intradermo-réactions) gardent une bonne valeur indicative. En cas de positivité, ils incitent à entreprendre la recherche d'IgE spécifiques par le RAST ou le test de dégranulation des basophiles humains (tab. 3). L'intolérance aux protéines du lait de vache (I.P.L.V.) du nourrisson bénéficie, de surcroît, du test de transformation lymphoblastique (TTL) et du test d'inhibition de migration leucocytaire (TIML). Les tests de provocation ont une place importante pour confirmer la réelle responsabilité de l'allergie mise en évidence, sur le plan biologique, dans les troubles cliniques. Enfin, l'élimination doit entraîner la guérison.

\section{CONCLUSION}

L'allergie aux protéines du lait revêt un renouveau d'actualité. La multiplicité des antigènes possibles, l'évolution variable et encore mal connue du pouvoir immunogène de chaque protéine en fonction de la digestion ou des traitements technologiques, posent certains problèmes au chimiste et à l'allergologue au niveau expérimental. 


\section{TABLEAU $3-T A B L E 3$}

Place de différents tests dans le diagnostic de l'allergie alimentaire au lait de vache

Usefulness prick tests, RAST, HBDT and challenges for diagnosis of intolerance to cow's milk proteins

\begin{tabular}{|c|c|c|c|}
\hline & Positif & Total des tests & Critère de positivité \\
\hline $\begin{array}{l}\text { Tests cutanés : } \\
\text { Prick } \\
\text { ID-R }\end{array}$ & $\begin{array}{r}1 \\
12\end{array}$ & $\begin{array}{r}8 \\
20\end{array}$ & $\begin{array}{l}\mathrm{d}_{0} \geqslant 5 \mathrm{~mm} \\
\mathrm{~d}_{0} \geqslant 10 \mathrm{~mm}\end{array}$ \\
\hline $\begin{array}{l}\text { Laboratoire : } \\
\text { TDBH } \\
\text { RAST }\end{array}$ & $\begin{array}{r}27 \\
4\end{array}$ & $\begin{array}{r}87 \\
9\end{array}$ & $\begin{array}{c}\text { dégranulation } \geqslant 30 \% \\
\text { (pour n basophiles } \\
\geqslant 30)\end{array}$ \\
\hline $\begin{array}{l}\text { Provocation : } \\
\text { SL } \\
\text { Natif } \\
\text { Inh. }\end{array}$ & $\begin{array}{l}2 \\
3 \\
1\end{array}$ & $\begin{array}{l}6 \\
9 \\
1\end{array}$ & $\begin{array}{l}\text { apparition des } \\
\text { symptômes }\end{array}$ \\
\hline
\end{tabular}

Prick = puncture épidermique.

$\mathrm{ID}-\mathrm{R}=$ intradermoréaction.

TDBH $=$ test de dégranulation des basophiles humains.

RAST $=$ radioallergo-sorbent-test.

$\mathrm{SL} \quad=$ sublingual.

Natif = aliment natif.

Inh. = inhalation.

Ces difficultés de base se croisent à celles qui naissent de tous les facteurs de variabilité, ayant trait à l'organisme : particularités du terrain allergique, de l'âge, de l'apport antigénique, des voies de sensibilisation, du rôle d'affections virales intercurrentes, de celui (protecteur) de l'allaitement maternel.

$\mathrm{Au}$ niveau du diagnostic, une méthodologie se dessine, fondée sur différents tests immunologiques, appuyés (chez le nourrisson) par l'étude de la muqueuse intestinale.

L'utilisation extensive des produits laitiers dans l'alimentation humaine, l'apparition de nouveaux produits issus de technologies actuelles, doivent rendre vigilants, de façon à être en mesure d'évaluer les possibles implications d'un pouvoir immunogène inattendu, sur la santé : soit qu'il apparaisse relié à une modification de la structure allergénique, soit qu'il soit en relation avec un haptène contaminant le lait. 


\section{Bibliographie}

ADRIAN (J.) (1972). - La réaction de Maillard vue sous l'angle nutritionnel. II. Comportement des matières alimentaires. Ind. Aliment. Agric., 12, 1713-1720.

Anderson (K. J.), Mac Laughlan (P.) and Coombs (R. R. A.) (1979). - Anaphylactic sensitivity of Guinea-pigs drinking different preparations of cows milks and infant formulae. Clin. Exp. Immunol., 35, 454-461.

BERRENS (L.) (1971). - The chemical classification of atopic allergens: an attemps at integration. Int. Arch. Allergy Appl. Immunol., 41, 186-198.

BleuminK (E.) and Young (E.) (1968). - Identification of the atopic allergen in cow's milk. Int. Arch. Allergy Appl. Immunol., 34, 521-534.

BleuminK (E.) (1970). - Food allergy. The chemical nature of the substances eliciting symptoms. World Rev. Nutr. Diet., 12, 505-570.

Campbell (S. G.) and Vier (E. J.) (1978). - The immunogenicity of milk from various species for the Guinea-pig. Clin. Exp. Immunol., 33, 514-518.

Deschamps (J. P.) (1978). - Allaitement maternel. Communication aux Journées "Alimentation de l'enfant normal ", Monaco, 23-26 avril.

Gérard (H.), Legras (B.) et Moneret-Vautrin (D. A.) (1981). - Le test de dégranulation des basophiles humains (T.D.B.H.). Intérêt d'une leucoconcentration et du calcul statistique appliqué au taux de dégranulation. Pathol. Biol., 29, 137-142.

Gunther (M.), Cheek (R.), Matthews (R. H.), Parish (W. E.) and Coombs (R.R.A.) (1960). - The level of antibodies to the proteins of cow's milk in the serum of normal human infants. Immunology, 3, 296-306.

Haddad (Z. H.), Kalra (V.) and Verma (S.) (1979). - - IgE antibodies to peptic and peptic-tryptic digests of betalactoglobulin: significance in food hypersensitivity. Ann. Allergy, 42, 368-371.

Hill (D. J.), Davidson (G. P.), Cameron (D. J. S.) and Barnes (G. L.) (1979). The spectrum of cow's milk allergy in childhood. Acta Paediatr. Scand., 68, $847-852$.

Kletter (B.), Gery (I.), Freier (S.) and Davies (A. M.) (1971). - Immune responses of normal infants to cow milk. I. Antibody type and kinetics of production. Int. Arch. Allergy Appl. Immunol., 40, 556-566.

Legrand (C.) et Paul (R.) (1977). - Produits lactés en forme de gel et leur procédé de fabrication. Brevets Français, $\mathrm{n}^{\circ} 2345936, \mathrm{n}^{\circ} 2345937$ et $\mathrm{n}^{\circ}$ 2347890 .

LippaRd (V. W.), Schloss (O. M.) and JoHNSON (P. A.) (1936). - Immune reactions induced in infants by intestinal absorption of incompletely digested cow's milk proteins. Am. J. Dis. Child., 51, 62-68.

Luquet (F. M.), Boudier (J. F.) et Vincent (J. P.) (1980). - Note à propos du vieillissement des poudres. Lait, 60, 295-297.

Mac Laughlan (P.), Anderson (K. J.) and Coombs (R. R. A.) (1981). - An oral screening procedure to determine the sensitizing capacity of infant feeding formulae. Clint. Allergy Appl. Immunol., 11, 311-318.

Moneret-Vautrin (D. A.) et Grilliat (J.P.) (1979). - Importance relative des divers constituants du lait dans l'allergie alimentaire. Cah. Nutr. Diét., 14, 237-240.

Moneret-Vautrin (D. A.) et Grilliat (J. P.) (1982). - Vraies et fausses allergies alimentaires. Rev. Prat., 32, 1537-1549. 
Moneret-Vautrin (D. A.), Pupil (P.), Courtine (D.) et Grilliat (J. P.) (1982). Asthme professionnel aux protéines du lactosérum. Communication au $4 \mathrm{e}$ Séminaire du G.R.E.P.A., Pont-à-Mousson, 6-7-8 mai.

PARISH (W. E.) (1982). - IgE anaphylactiques (IgG-STS) dans l'allergie alimen-

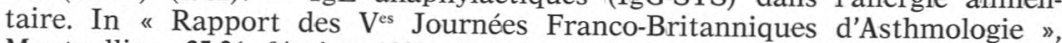
Montpellier, 25-26 février 1981, Ed. by Laboratoires Boehringer-Ingelheim, Reims.

Scheinmann (P.), Gendrel (D.), Charlas (J.) and Paupe (J.) (1976). - Value of lymphoblast transformation test in cow's milk protein intestinal intolerance. Clin. Allergy, 6, 515-521.

WAHL (J. M.) (1980). - Enzymatic unmasking for antibodies of penicilloyl residues bound to albumin. Biochem. Pharmacol., 29, 195-199.

Wahn (U.), Peters (T.) and Siraganian (R. P.) (1981). - Allergenic and antigenic properties of bovine serum albumin. Mol. Immunol., 18, 19-28.

Wright (R. N.) and Rothberg (R.M.) (1971). - The reactions of pepsin and tryptic digestion products if bovine serum albumin with antisera from rabbits ingesting this protein. J. Immunol., 107, 1410-1418. 